\title{
Comparative Study of Women's Self-Reporting Stress during Pregnancy and Post-Partum across Disparate Immigrant and Socioeconomic Strata
}

\author{
Hoskins IA, MD, FACOG, FACS ${ }^{1 *}$ and Cerfolio N, MD, FACLP, FAPA ${ }^{2}$ \\ ${ }^{1}$ Department of Ob/Gyn, NYU School of Medicine, New York, NY, USA \\ ${ }^{2}$ Department of Psychiatry, Mt. Sinai Icahn School of Medicine, New York, NY, USA \\ *Corresponding author: Hoskins IA, Department of Ob/Gyn, NYU School of Medicine, New York, NY, USA
}

Received: August 09, 2021; Accepted: August 16, 2021; Published: August 20, 2021

\section{Introduction}

Women experience many different types of stresses during their lifetimes. Child bearing is a known life event that although often considered joyous, may be a stressful time in a woman's life. Given that approximately $50 \%$ of pregnancies are unplanned, this is especially applicable to recent immigrants because of the known additional stresses such as language and cultural barriers. In these situations, the pregnant women may perceive variable and/or unavailable support from family and/or friends.

Psychological stress is known to have negative effects on maternal mental health, and may result in adverse outcomes such as depression and anxiety [1,2]. Most studies assessing effects of stress on pregnancy outcomes are nonspecific and confusing. Cohen et al. [3] described the results of a Perceived Stress Scale which was administered to different groups based on gender, race, age, demographics, and income. Women, regardless of income, were more likely to score high and describe feeling stressed. Alderdice et al. [4] describe the results of a Prenatal Distress Questionnaire, which was administered to 263 low risk pregnant women during the second trimester. Only one of the top three factors of concern pertained to worry about care for baby after birth. The other two factors were about concerns regarding physical effects of pregnancy, specifically weight gain, nausea, vomiting, swelling and backache.

\section{Objective}

To study whether women from disparate cultural, immigrant and Socioeconomic (SEC) strata have differences in their self-perceptions of stress levels during pregnancy and post-partum.

\section{Materials and Methods}

Patients receiving care from a "Non-Private" clinic in an inner city Hospital, serving indigent Chinese recent immigrants, with low Socioeconomic [SEC] status and language/cultural barriers were recruited for the study. Their outcomes were compared to those from patients who were seen in a "Private" clinic setting. These women had higher SEC status, higher levels of education, were employed in the financial industry in a large metropolitan area, and had minimal to no language/cultural barriers.
Each patient completed a questionnaire which was adapted from American Psychiatric Association guidelines and assessed selfperceived stress (Figure 1). The answers were scored from 1 to 5 , with 1 being the highest level of coping, thus reflecting a low stress level. The lowest level of coping, which represented a high stress level, was assigned a score of 5 . Answers that received a score of $>/=3$ were classified as "poor coping/high stress". All patients completed the questionnaires during the first and third trimesters, and at the postpartum visit. Although the questionnaire was in English, translation services were available for the patients who requested it.

The first and third trimesters of pregnancy were chosen because the assessments were intended to assess the patients' excitement or apprehension towards childbirth and becoming a parent. The postpartum questionnaire was intended to assess the reality of the baby's arrival, including mother's sleep deprivation and concerns about parenting/coping.

Statistical analysis of the outcomes in the 2 Groups was by Chi square test. Statistical significance was a $p$ value of $<0.05$.

\section{Results}

During the study period of one year, 3192 women with low risk, singleton pregnancies were enrolled into the study. All reported the presence of a partner/support person in their lives.

Of these, 487 subjects were recruited from the "Non-Private" clinics and 519 women were recruited from the "Private" clinics.

The questionnaires were completed by 1006 patients (58\%) at the 3 study points, i.e. $1^{\text {st }}$ and $3^{\text {rd }}$ trimesters and at the post-partum visit. The patients could self-select to remain anonymous if they so desired, as this was unlikely to affect the outcomes.

The results of the demographics are shown in Table 1. There were statistically significantly increased patients of Chinese ethnicity in Group I (recent immigrants, low SEC status and language/cultural barriers). There were no significant differences between the two Groups regarding maternal age, gestational age at delivery, and neonatal birth weight. 
Hoskins IA (2021) Comparative Study of Women's Self-Reporting Stress during Pregnancy and Post-Partum across Disparate Immigrant and

Socioeconomic Strata

\section{Questionnaire}

Please answer all questions below.

Please pick only one option for each question.

( 1 = high coping/low stress; 5 = low coping/high stress).

Thank You.

High Coping/Low Stress

12

\section{Low coping/High Stress}

345

1. How are you coping with your pregnancy?

2. How do the physical effects of your pregnancy affect your coping ability?

3. How would you rate your mood in relation to your pregnancy?

4. What are the effects of your pregnancy on your ability to perform daily tasks?

5. What are the effects of your pregnancy on your personal relationships?

6. Describe the degree to which you are concerned about the pregnancy on your future health.

Comments (optional):

Positive:

Negative

Name: (optional):

Clinic Location:

$1^{\text {st }}$ Trimester $3^{\text {rd }}$ trimester Post-partum

Figure 1: Questionnaire.

Table 1: Demographic Data.

\begin{tabular}{|c|c|c|}
\hline & Group 1 & Group 2 \\
\hline & \multicolumn{2}{|c|}{$\mathrm{N}=1006$} \\
\hline & $\begin{array}{c}\text { Non-Private Clinic } \\
\mathrm{N}=487\end{array}$ & $\begin{array}{c}\text { Private Clinic } \\
\mathrm{N}=519\end{array}$ \\
\hline Age (years) & $25 \pm 3 y$ & $31 \pm 2 y$ \\
\hline Gestational age at delivery & $39 \pm 2$ weeks & $38 \pm 2$ weeks \\
\hline \multicolumn{3}{|l|}{ Race } \\
\hline Caucasian & $9(1.8 \%)$ & $269(51.8 \%)$ \\
\hline Hispanic & $21(4.3 \%)$ & $86(16.6 \%)$ \\
\hline African American & $2(0.4 \%)$ & $61(11.8 \%)$ \\
\hline Chinese $^{*}$ & $455(93.4 \%)$ & $61(11.8 \%)$ \\
\hline Parity & 1.3 & 1.1 \\
\hline High School Diploma* & $236(48.5 \%)$ & $504(97.1 \%)$ \\
\hline English Fluency* & $200(41 \%)$ & $519(100 \%)$ \\
\hline Neonatal Weight & $3396 \pm 241 \mathrm{~g}$ & $3511 \pm 331 \mathrm{~g}$ \\
\hline
\end{tabular}

${ }^{*} \mathrm{P}<0.05$.

The results of the self-reporting of moderate/high stress scores are shown in Table 2. Although the majority of patients in both Groups self-reported low coping/high stress scores $(>/=3)$ during the $1^{\text {st }}$ and $3^{\text {rd }}$ trimesters, there were more women in Group I who reported these findings, $67 \%$ and $63 \%$ in both the trimesters, versus $59 \%$ and $56 \%$ of the women in Group 2 reporting the same. This was not statistically
Table 2: Results of the Questionnaires for the 2 trimesters and Post-Partum: Moderate/ High Stress $(>/=3)$.

\begin{tabular}{|l|c|c|}
\hline & Group 1 & Group 2 \\
\hline & $\begin{array}{c}\text { Non-Private Clinic } \\
\mathbf{N}=\mathbf{4 8 7}\end{array}$ & $\begin{array}{c}\text { Private Clinic } \\
\mathbf{N}=\mathbf{5 1 9}\end{array}$ \\
\hline $1^{\text {st }}$ Trimester & $67 \%$ & $59 \%$ \\
\hline $3^{\text {rd }}$ Trimester & $63 \%$ & $56 \%$ \\
\hline Post-Partum & $19 \% *$ & $71 \%^{*}$ \\
\hline
\end{tabular}

${ }^{*} \mathrm{P}<0.05$.

significant. During the post-partum period, only $19 \%$ of the women in Group 1 self-reported scores $>/=3$ (low coping, high stress), versus those in Group 2, where $71 \%$ reported scores $>/=3$, reflecting low coping/high stress. This was statistically significant.

Only $13.2 \%$ of the optional comments submitted by the women were positive, with statements such as "pregnancy is easier than I thought, work is not a problem, my partner/support person is very helpful". These findings were similar in both Groups.

\section{Conclusions}

In general, most attempts at assessing the effects of stress on pregnancy across diverse ethnicities and socio-economic strata have revealed mixed results. Cohen et al. [3] reported that stress was higher in women versus men and increased with decreasing age, education and income. However, these women were not selected for pregnancy. 
Our questionnaire allowed a comparison of self-reported stress in women with disparate SEC and immigrant status, and across the duration of the pregnancy.

The patients in Group 1 who accessed healthcare from the "Non Private" Clinic were more likely to see multiple providers (Attendings, Residents, Fellows and Allied Health Personnel), which may have adversely affected the continuity of care that was provided and may have resulted in the patients' describing low coping/high stress levels during the pregnancy.

The patients in Group 2, accessed healthcare from the "Private" Clinic and saw the same clinicians throughout, thus enhancing continuity of care and allowing improved communication and relationships with the providers. This may have resulted in more of these women describing high coping/low stress levels.

Interestingly, equivalent numbers of patients across both Groups attributed their stress levels and coping abilities to the work/life stresses versus their SEC or immigrant status and any language/ cultural barriers.

During the postpartum period, only 19\% of the women in Group 1 continued to express low coping/high stress scores. We hypothesize that, in this predominantly Chinese population of recent immigrants, the availability of cultural support systems (family, friends, neighbours) within that community likely provided help to them regarding child rearing and post-partum recovery issues.

Conversely, $71 \%$ of the women in Group 2, reported low coping/ high stress scores during the postpartum period. We hypothesize that this may be due to the absence of support systems (family, friends, neighbours), thus leaving the burdens of child rearing and post-partum recovery on the women themselves. This caused their stress levels to remain high and was similar to the levels noted during the pregnancy.

\section{Discussion}

Our knowledge of women's coping/stress levels during pregnancy and postpartum in various ethnic, culturally diverse and disparate SEC groups is limited. The concept of a "Sense of Coherence" (SOC), also called salutogenesis by Antonovsky [5] reflects how people manage stress and stay well and thus plays an essential role in a person's satisfaction with life events. SOC is based on three components, ability to understand (comprehensibility), ability to control (manageability), and significance (meaningfulness). The more evident these feelings are, the higher a person's SOC. It is hypothesized that a high SOC is a prerequisite for successfully coping with a stressful situation, leading to better well-being.

Sjostrom et al. [6] used a SOC scale to study pregnant women's self-perception of well-being during pregnancy and postpartum. Of the 120 participants who completed the survey, women who were in the third trimester gave significantly higher scores $(>/=3)$, reflecting increased stress during this period versus their results during the first trimester. When the SOC ratings were high, the women's sense of wellbeing was also high and they reported less anxiety and depression regarding the pregnancy. Although their subjects were of a uniform ethnicity (Scandinavian) and included complicated pregnancies, the findings were similar to those of our study.
During the postpartum period however, women in Group 2 continued to report low coping/high stress levels versus those in Group 1, whose low coping/high stress levels had markedly decreased. This was contrary to the findings by Sjogstrom et al. who found that high SOC levels (reflecting high coping/low stress) during the first and third trimesters, also predicted similar results in the postpartum period.

Other factors that are well known to affect mental status and mood, such as work load, substance and emotional, psychological, physical abuses [6] were not assessed in this study. The focus of our study was mainly to assess the women's self-perception of coping/ stress levels during pregnancy and postpartum in disparate ethnic and SEC groups.

Another potentially important factor of whether the pregnancy was planned or not, was also not addressed in our study. The adverse impact of an unintended pregnancy regardless of SEC and immigrant status, education level, and cultural/language barriers, on a woman's coping/stress level is well known [7]. That confounding variable was not addressed in this study.

Our findings may have future perinatal implications for the wellbeing of mothers and their unborn babies by helping to address and promote the need for mental health support and thus decrease known maternal stress related complications such as preterm delivery, small for gestational age and intrauterine growth restriction [8]. Larger studies are needed in order to better understand coping/stress levels in women with disparate ethnicities and SEC strata during these very important life events.

\section{References}

1. ACOG Committee Opinion \#630 (2015) Screening for Perinatal Depression. Obstetrics and Gynecology 125: 1268-1271.

2. Swanson LM, Pickett SM, Flynn H, Armitage R (2011) Relationships among depression, anxiety and insomnia symptoms in perinatal women seeking mental health treatment. Journal of Women's Health 20: 553-538. [crossref]

3. Cohen S, Janicki-Deverts D (2012) Who's stressed? Distributions of Psychological Stress in the US in Probability Samples from 1983, 2006, and 2009. J of Applied Social Psychology 42: 1320-1334.

4. Alderdice F, Lynn F (2011) Factor structure of the Prenatal Distress Questionnaire. Science Direct 27: 553-559. [crossref]

5. Antonovsky A (1979) Health, Stress and Coping. San Francisco. Jossey-Bass Publishers.

6. Sjostrom H, Langius E, Klof A (2004) Wellbeing and sense of coherence during pregnancy. Acta Obstet Gynecol Scand 83: 1112-1118. [crossref]

7. Russo NF (2014) Abortion, unwanted childbearing and mental health. Salud Mental 37: Julio-Augosto.

8. Peacock JL, Bland JM, Anderson HR (1995) Preterm delivery: effects of socioeconomic factors, psychological stress, smoking, alcohol and caffeine. BMJ 311: 531-535. [crossref]

\section{Citation:}

Hoskins IA, Cerfolio N (2021) Comparative Study of Women's Self-Reporting Stress during Pregnancy and Post-Partum across Disparate Immigrant and Socioeconomic Strata. Integr Gyn Obstet J Volume 4(2): 1-3. 